\title{
Myeloid progenitors differentiate into microglia and promote vascular repair in a model of ischemic retinopathy
}

\author{
Matthew R. Ritter, ${ }^{1}$ Eyal Banin,,1,2 Stacey K. Moreno, ${ }^{1}$ Edith Aguilar, ${ }^{1}$ \\ Michael I. Dorrell, ${ }^{1}$ and Martin Friedlander ${ }^{1}$ \\ ${ }^{1}$ Department of Cell Biology, The Scripps Research Institute, La Jolla, California, USA. \\ 2Department of Ophthalmology, Hadassah-Hebrew University Medical Center, Jerusalem, Israel.
}

\begin{abstract}
Vision loss associated with ischemic diseases such as retinopathy of prematurity and diabetic retinopathy are often due to retinal neovascularization. While significant progress has been made in the development of compounds useful for the treatment of abnormal vascular permeability and proliferation, such therapies do not address the underlying hypoxia that stimulates the observed vascular growth. Using a model of oxygen-induced retinopathy, we demonstrate that a population of adult BM-derived myeloid progenitor cells migrated to avascular regions of the retina, differentiated into microglia, and facilitated normalization of the vasculature. Myeloid-specific hypoxia-inducible factor $1 \alpha(\mathrm{HIF}-1 \alpha)$ expression was required for this function, and we also demonstrate that endogenous microglia participated in retinal vascularization. These findings suggest what we believe to be a novel therapeutic approach for the treatment of ischemic retinopathies that promotes vascular repair rather than destruction.
\end{abstract}

\section{Introduction}

The majority of eye diseases that lead to vision loss do so as a result of abnormal blood vessel growth, often in response to retinal ischemia (1). When ischemic conditions develop in other organs and tissues, such as the heart or brain, growth of collateral vessels may be beneficial. However, in the eye these vessels leak fluid and bleed, causing vision loss secondary to retinal edema, hemorrhage, and/or fibrovascular proliferation (2). Historically, interventions to treat these conditions have been designed to prevent the growth of new vessels or to close, ablate, or remove abnormal vessels that have already formed. Laser photocoagulation has been used to destroy ischemic retinal tissue with the idea of causing the regression of neovascularization as the ischemic drive is decreased. More recently, advances in the field of angiogenesis have led to the development of many antiangiogenic compounds such as VEGF inhibitors, angiostatic steroids, integrin antagonists, and others (3-7). While alignment of supply and demand through laser ablation of ischemic retina is efficacious in certain subgroups of ischemic retinopathies, this approach and the use of angiostatic compounds often fail to completely inhibit abnormal vascular growth and are associated with a large degree of tissue injury and the risk of local, as well as systemic, complications (8).

In the present study, we suggest an alternative approach to the problem of ischemia-induced neovascularization in the eye; rather than prevent or eliminate the neovasculature, we propose to improve and stabilize the vascular response to hypoxia. We have previously shown that lineage-negative HSCs (Lin-HSCs) target

Nonstandard abbreviations used: EPC, endothelial progenitor cell; ERG, electroretinography; GS lectin, isolectin GS-IB 4 ; HIF- $1 \alpha$, hypoxia-inducible factor $1 \alpha$; Lin-HSC, lineage-negative HSC; OIR, oxygen-induced retinopathy; ROP, retinopathy of prematurity.

Conflict of interest: The authors have declared that no conflict of interest exists. Citation for this article: J. Clin. Invest. 116:3266-3276 (2006). doi:10.1172/JCI29683. activated astrocytes in the developing mouse retina and participate in normal and pathologic angiogenesis (9-11). Furthermore, when these cells were injected into the eyes of neonatal $r d / r d$ mice, which have inherited retinal degeneration, a rescue effect was observed in the vasculature as well as in the neural retina, both of which ordinarily degenerate rapidly after birth. A large body of work suggests that BM-derived progenitors may have beneficial effects in ischemic hearts and limbs, but the precise cellular mechanisms involved remain unclear $(12,13)$. Our previous findings that BM-derived progenitors can exert effects on both developing and degenerating retinal vessels has prompted our study of their possible role in ischemic retinopathies, using the oxygeninduced retinopathy (OIR) mouse model (14). We show here that transplanted BM-derived progenitors dramatically accelerated retinal vascular repair of OIR with no observed long-term toxicity, increasing the rate of physiological intraretinal revascularization while at the same time markedly reducing the formation of abnormal, preretinal neovascularization. We defined an active myeloid population that mediated this effect and demonstrated that the transplanted myeloid progenitors differentiated into cells with characteristics of microglia following their injection into the vitreous of mice. The molecular basis of these findings was further investigated using BM from mice with targeted deletions of hypoxia-inducible factor $1 \alpha$ (HIF-1 $\alpha$ ) in cells of the myeloid lineage, which demonstrated that the HIF- $1 \alpha$ transcription factor was required for this population of BM cells to mediate the observed trophic repair. In this report we also established a role for microglia in regulating retinal vascularization during development by demonstrating that depletion of retinal microglia profoundly inhibited vessel growth and patterning. These results suggest what we believe to be a novel approach to the treatment of ischemic retinopathies that involves the use of autologous adult BM-derived progenitor cells to rebuild and stabilize functional vasculature in hypoxic retinal tissue, rather than to eliminate the problematic vessels that result. 


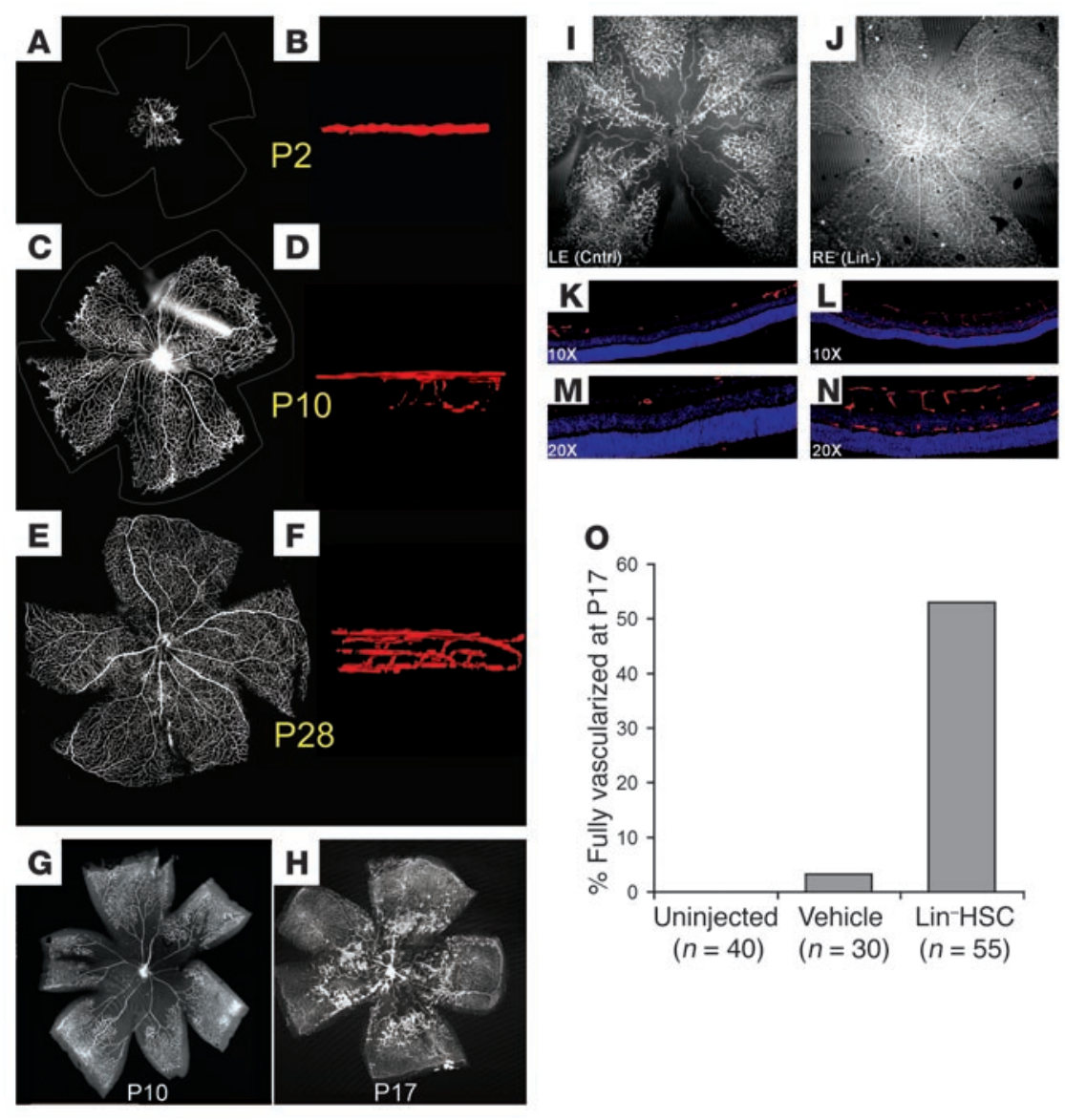

Figure 1

Retinal vascular development in normal and OIR mice. The mouse is born with a largely avascular retina (A and $\mathbf{B})$. During the first postnatal week, superficial retinal vessels grow radially from the optic nerve head $(\mathbf{C})$. The deep retinal vasculature is established through branching of the superficial layer during the second week (D). A third intermediate plexus of vessels forms, and the mature retinal vasculature is established at around P30 (E and $\mathbf{F})$. Exposure to hyperoxia causes central vaso-obliteration (G), and after returning to normoxia at $\mathrm{P} 12$, characteristic preretinal neovascular tufts form at the interface between the vascularized (peripheral) and avascular (central) retina (H). (I-N) Lin-HSCs promote vascular repair in the OIR model. Lin-HSC injected intravitreally prior to oxygen exposure dramatically accelerated revascularization compared with the vehicle-treated fellow eye at P17. While retinas treated with vehicle showed partial absence of the superficial vasculature (I) and complete absence of the deep retinal vasculature ( $\mathrm{K}$ and $\mathbf{M})$, the Lin-HSC-treated eye showed relatively normal retinal vasculature $(\mathbf{J}, \mathbf{L}$, and $\mathbf{N})$. ( $\mathbf{0}$ ) A dramatically higher proportion of eyes treated with Lin-HSC were fully revascularized at P17 compared with control eyes. Vessels were visualized by cardiac perfusion of fluorescein-dextran in $\mathbf{A}-\mathbf{F}, \mathbf{I}$, and $\mathbf{J}(\mathbf{B}, \mathbf{D}$, and $\mathbf{F}$ are images taken from $3 \mathrm{D}$ renderings rotated 90 degrees), and by GS lectin in $\mathbf{G}, \mathbf{H}$, and $\mathbf{K}-\mathbf{N}$. Nuclei in $\mathbf{K}-\mathbf{N}$ were labeled with DAPI (blue). RE, right eye; LE, left eye. Magnification, $\times 4(\mathbf{A}, \mathbf{C}, \mathbf{E}, \mathbf{G}$, and $\mathbf{H}), \times 10(\mathbf{I}$ and $\mathbf{J}), \times 60$ (B, D, and F).

\section{Results}

Retinal vascular development and the OIR mouse model. Normal retinal vascular development in postnatal mice grown under normoxic conditions is shown in Figure 1, A-F. At P2 only budding superficial vessels were observed occupying a single plane around the optic disc (Figure 1, A and B). Over the course of the next week, the primary superficial network extended toward the periphery, reaching the far periphery at approximately P12 (Figure 1C). Between P7 and P12, the secondary (deep) plexus developed (Figure 1D), and during the third week, the tertiary (intermediate) layer of vessels formed. This was followed by the remodeling of the fully vascularized retina (Figure $1 \mathrm{E}$ ) and the establishment of an adult vasculature by the end of the first month (Figure 1F) $(15,16)$.

In contrast, exposure to $75 \%$ oxygen from P7 to P12 severely disrupted the normal development of the retinal vasculature. Marked regression of the superficial network of vessels that had already formed in the central retina occurred, especially along the arteries (Figure 1, G and H), and development of the deep plexus was significantly delayed. When the mice returned to normoxia at P12, relative hypoxia ensued in the severely hypovascular central retina, causing abnormal vascular growth. At P17, abnormal preretinal neovascular tufts were seen in the midperiphery, at the interface between the hypovascular central retina and the more vascularized periphery (Figure $1 \mathrm{H})$. These neovascular tufts protruded above the inner limiting membrane of the retina into the vitreous and often persisted until P21 or later.
Injection of BM subpopulations promotes retinal vascular repair. In order to evaluate the possible effect of BM-derived Lin ${ }^{-} \mathrm{HSCs}$ in a model of retinal ischemia, we transplanted these cells into the eyes of mice subjected to OIR. We have recently developed a method of quantifying both vascular obliteration and preretinal neovascular tuft formation in the same retina using confocal microscopy and digital image analysis (17). A single injection of Lin-HSCs, but not vehicle alone or control cells, performed between P2 and P7 dramatically altered the recovery of the retinal vasculature following oxygen exposure (Figure 1, J, L, N, and $\mathrm{O}$, and Figure 2, A-F). In over $50 \%$ of the eyes examined, fully developed superficial and deep retinal vasculature was observed at P17 in Lin-HSC-injected eyes, while contralateral vehicle-injected eyes exhibited persistent, large avascular areas and few or no deep vessels (Figure 1, I-O). The use of paired eyes in the same animal (e.g., right, experimental; left, control) provided a biologically and statistically powerful comparison that effectively equalized most genetic and environmental variables.

The area of vascular obliteration measured at P17 was significantly reduced by over $75 \%$ in Lin-HSC-treated retinas compared with eyes receiving vehicle or no injection (Figure 2E). Similarly, eyes treated with Lin-HSCs had an approximately $70 \%$ reduction in preretinal neovascular tuft area compared with vehicle-injected eyes and greater than $80 \%$ reduction versus noninjected controls (Figure 2F). Thus, Lin-HSC treatment had a dramatic effect on the 2 major parameters of the mouse OIR model, simultaneously reducing formation of neovascular tufts while accelerating physi- 

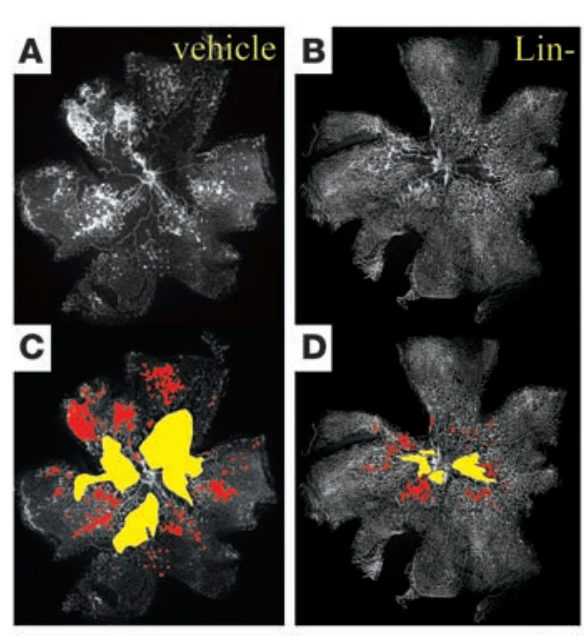

\section{E}
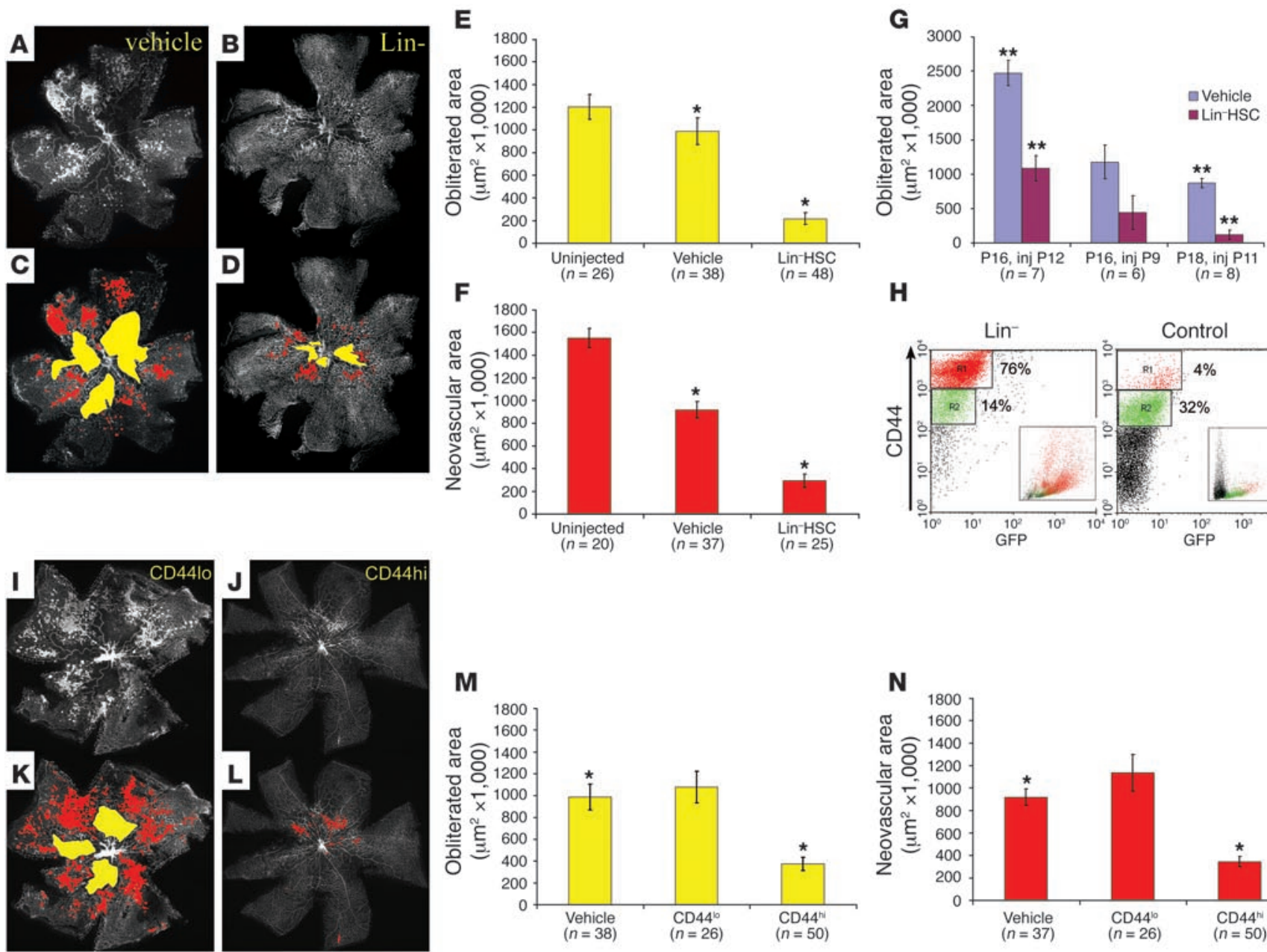

H
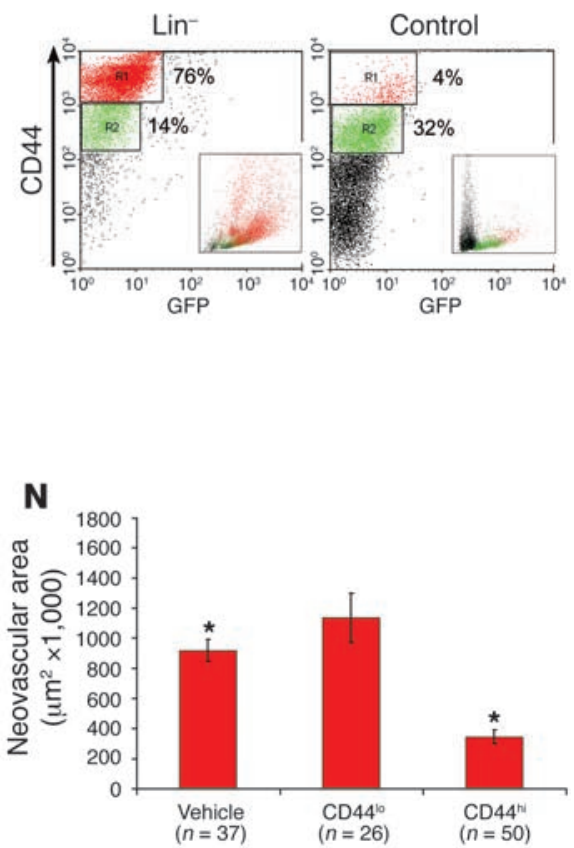

Figure 2

BM subpopulations accelerate retinal revascularization and reduce preretinal neovascular tuft formation in OIR. (A-D) Computer-assisted image analysis was used to calculate the area of retinal vessel obliteration (yellow) and preretinal neovascular tuft formation (red) in whole mounts from OIR eyes at P17 (17). (E) Retinas treated with Lin-HSC prior to hyperoxia showed an almost 6-fold reduction in obliterated area versus uninjected controls and an approximately 5-fold reduction compared with vehicle-treated controls. (F) Lin-HSC treatment significantly reduced neovascular tufts compared with uninjected and vehicle-treated eyes. (G) Lin-HSCs reduced obliteration when administered prior to hyperoxia and during hyperoxia (P9 or P11) or just after return to normoxia (P12). inj, injected. (H) Mouse BM contained CD44hi and CD44lo fractions and the Lin-HSC population was enriched for CD44 ${ }^{\text {hi }}$ cells (red). Insets: Light-scattering properties of the CD44 ${ }^{\text {hi }}$ cells were typical of monocytes and granulocytes, while those of CD44/o cells were typical of lymphocytes. (I and J) Representative P17 retinas from eyes treated with CD44lo and CD44hi BM cells at P7. (K and L) Areas of obliteration (yellow) and neovascularization (red) at P17. When treated at P7, vascular obliteration (M) and neovascularization $(\mathbf{N})$ were reduced in eyes treated with $\mathrm{CD} 44^{\mathrm{hi}}$ cells with efficacy similar to eyes treated with Lin-HSC cells. Obliteration was similar between eyes treated with Lin-HSC and CD44hi cells, and neovascularization did not differ significantly between these groups $(P=0.25)$. Values represent mean \pm SEM. ${ }^{*} P<10^{-5} ;{ }^{*} P \leq 0.006$. Magnification, $\times 4$.

ologic revascularization of the central retina. It is important to point out that cell treatment did not prevent the vaso-obliteration that occurred during exposure to high oxygen levels between P7 and P12. Rather, the effect of BM cell treatment was in accelerating vascular repair and normalization after return to room air.

The experiments described thus far involved single injections performed between P2 and P7, prior to exposure to hyperoxia. To determine whether Lin-HSCs could also affect vascular repair if injected later, either during the hyperoxia phase of the cycle or upon return to normoxia, injections were performed at P9, P11, or $\mathrm{P} 12$, and retinas were evaluated at various later time points. These experiments demonstrated that injection of Lin-HSCs effectively accelerated the repair of all vascular layers and reduced the area of obliteration even when administered during hyperoxia or upon return to normoxia (Figure $2 \mathrm{G}$ ).

To address the issue of possible long-term effects of injected cells on the retina, we examined retinal structure (by histology) and function (by electroretinography [ERG]) up to 6 months after transplantation. In histologic sections, teratomas or tumors were not observed in any of the eyes. We did not find significant differences in the thickness of the retina or different retinal layers between $\mathrm{Lin}^{-} \mathrm{HSC}$-treated eyes and various controls. Dark-adapted rod b-wave amplitudes, mixed cone-rod aand b-wave amplitudes, and light-adapted cone ERG responses 


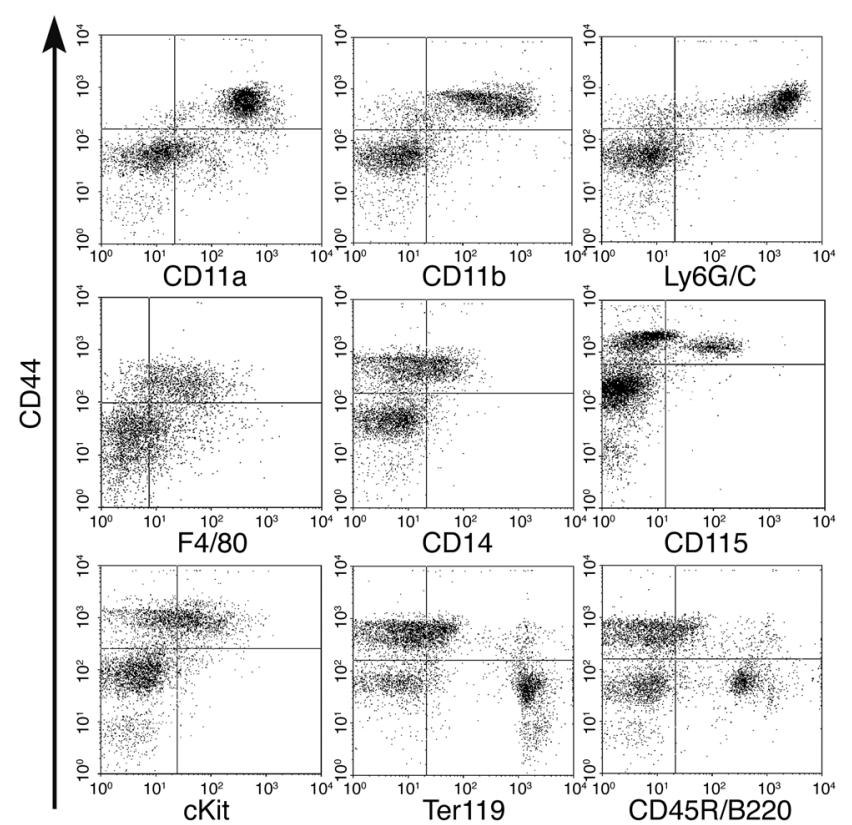

Figure 3

The CD44 ${ }^{\text {hi }}$ subpopulation expresses markers and genes characteristic of myeloid cells. Two-color flow cytometry was used to characterize CD44 populations in whole mouse BM. All cells were labeled with an antibody against CD44 and colabeled with the indicated antibodies. CD44 hi cells are distinguished from CD44 ${ }^{\text {lo }}$ and CD44- cells by the horizontal lines in each plot. The CD44hi population showed strong labeling for CD11b and Ly6G/C. Fractions of CD44hi cells (to the right of the vertical lines) were positive for F4/80, CD14, and CD115. These antigens are present on cells of the myeloid lineage (38-42). Positive labeling for CD11a and cKit was also observed on CD44hi cells. CD44lo cells labeled strongly with Ter119 and CD45R/B220 which are markers for erythroid and lymphoid cells, respectively $(43,44)$.

at the age of 4-6 months did not differ among room air-grown active cell population-treated OIR eyes or OIR controls.

Positive selection of an active population using CD44. In an effort to better characterize the active cell population(s) and to understand the underlying repair mechanisms, we sought to identify a single marker that could be used to isolate active cells from the BM. Using flow cytometry, we screened a large panel of hematopoietic progenitor and mature cell markers and compared their expression in the active Lin-HSCs versus that in control BM cells, which were previously shown to be inactive in other experimental systems $(9,10)$. One of the candidate markers, CD44 (a hyaluronic acid receptor), proved to be differentially expressed in these 2 populations. Labeling for CD44 produced 2 distinct positive populations: one with high expression of this marker $\left(C D 44^{\text {hi }}\right)$ and the other with lower levels of expression (CD44 $\left.{ }^{\text {lo }}\right)$. $\mathrm{CD} 44^{\mathrm{hi}}$ cells were present in a significantly higher proportion of the Lin-HSCs (76\%) than in the control BM cell population (4\%) (Figure $2 \mathrm{H}$ ). This distribution suggested that CD44hi cells were reasonable candidates for further study.

CD $44^{\text {hi }}$ cells were tested in the OIR model for their ability to facilitate vascular repair. Using the same experimental design as that described for Lin-HSC injections, we demonstrated that CD44hi cells injected at P7 promoted retinal vascular repair in this model with efficacy similar to that observed with Lin-HSCs (Figure 2,
$\mathrm{J}$ and $\mathrm{L}-\mathrm{N})$. In contrast, $\mathrm{CD} 44^{\mathrm{lo}}$ cells, which were rarely found in the retina, had no positive effect on repair (Figure 2, I, K, M, and N). CD44hi cells, when compared with vehicle, were also shown to significantly reduce pathological neovascularization by $52 \%$ when injected at P15, after the neovascular phase had begun $(n=6$, $P=0.008)$. An $18 \%$ reduction in obliterated area was also observed $(P=0.052)$. Based on these findings, we were able to use CD44 expression to positively select a highly purified active population from whole BM using a single marker, thus facilitating further analysis of the active cell population(s).

CD44 ${ }^{\text {bi }}$ cells express genes and markers characteristic of myeloid cells. Detailed characterization of the CD44hi population was performed by flow cytometry and large-scale expression analysis (Figure 3 and Table 1). Both approaches revealed that CD44hi cells expressed many markers consistent with myeloid origin. Strong expression of CD11b (Mac1) and Ly6G/C (Gr1) was observed on these cells at the protein level, while less intense positive labeling was detected for F4/80, CD14, and CD115 (Figure 3). The mRNA of several myeloid-specific genes including CD204, CD114, CD33, and CD115 was found to be highly expressed in CD $44^{\text {hi }}$ compared with CD44 ${ }^{\text {lo }}$ cells (Table 1). In contrast, at the protein level, the CD $44^{\text {lo }}$ population had significantly higher expression of Ter119 and CD45R/B220, which are markers of erythroid and B cells, respectively (Figure 3). Using gene expression array analysis, a number of genes associated with lymphoid cells, including CD19, CD79a, and CD22, were highly expressed in CD44 ${ }^{\text {lo }}$ compared with CD $44^{\text {hi }}$ cells (Table 1). Thus, analysis at both the mRNA and protein levels identified the active CD $44^{\text {hi }}$ population as primarily myeloid in origin, while the inactive CD44 ${ }^{\text {lo }}$ cells were identified as erythroid and lymphoid cells.

$H I F-1 \alpha$ expression is required for myeloid cell-mediated effects in the OIR model. We next sought to better understand the molecular mechanisms underlying the observed vascular repair mediated by transplanted myeloid progenitors. We reasoned that hypoxia would have a prominent role in this system and thus chose to investigate the role of HIF- $1 \alpha$, a well-studied modulator of cellular response to low oxygen conditions and regulator of angiogenic gene expression (18). Targeted deletions of the HIF-1 $\alpha$ transcription factor were created via crosses into a background of cre expression driven by the lysozyme M promoter (lysMcre) $(19,20)$, which allows specific deletion of the factor in the myeloid lineage. We isolated CD44hi myeloid progenitors from the BM of these mice and tested their ability to promote vascular repair in the OIR model compared with wild-type CD44hi cells when transplanted at P7. Importantly, we found no differences between the CD44 ${ }^{\text {hi }}$ cells isolated from the myeloid-specific HIF- $1 \alpha$ knockout strain and the wild-type strain in terms of surface marker expression or light scattering properties. However, the HIF-1 $\alpha$-deficient cells failed to promote repair, while the wild-type cells dramatically accelerated vascular repair, as described above (Figure 4). As it is likely that the accelerated repair observed here relies on more than a single factor, HIF- $1 \alpha$ is a reasonable choice for study because it regulates the transcription of numerous genes that could conceivably have roles, including VEGF, IGFs, TGF- $\beta$, and others (18). In addition, this finding strongly supports the concept that myeloid cells constitute the active population mediating vascular repair, since suppression of HIF-1 $\alpha$ gene expression in this knockout was specific for cells of the myeloid lineage and all other BM-derived cells are expected to retain normal activity. 
Table 1

Large-scale expression analysis of BM populations

\begin{tabular}{|c|c|c|c|c|c|}
\hline & CD44 ${ }^{\text {hi }}$ & CD44 ${ }^{10}$ & Ratio & GenBank ID & Expressed by \\
\hline \multicolumn{6}{|c|}{ Higher expression in CD44 $4^{\text {hi }} B M$ cells } \\
\hline CD204 & 117.5 & 0.4 & 293.8 & BC003814 & Macrophages \\
\hline CD114 & $1,079.5$ & 12.2 & 88.5 & NM_007782 & Neutrophils, granulocytes \\
\hline CD33 & $2,006.3$ & 23.2 & 86.5 & NM_021293 & Myeloid progenitors \\
\hline CD115 & $2,412.3$ & 72.6 & 33.2 & Al323359 & Monocytic progenitors \\
\hline CD34 & 956.5 & 74.9 & 12.8 & NM_133654 & Hematopoietic precursors, ECs, BM stroma, mast cells \\
\hline CD9 & $1,072.7$ & 115.8 & 9.3 & NM_007657 & Myeloid cells, activated T Iymphocytes, subset of B lymphocytes \\
\hline CD68 & 783.6 & 101.1 & 7.8 & BC021637 & Tissue macrophages and DCs \\
\hline CD4 & 126.8 & 18.1 & 7.0 & NM_013488 & DCs, subset of thymocytes and T lymphocytes \\
\hline CD80 & 92.8 & 17.2 & 5.4 & AA596883 & Monocytes, macrophages, DCs, activated T and B lymphocytes \\
\hline CD44 & $3,497.9$ & 679.6 & 5.1 & AW146109 & Broad expression \\
\hline \multicolumn{6}{|c|}{ Higher expression in CD44/0 BM cells } \\
\hline CD19 & 9.5 & $4,268.3$ & 449.3 & NM_009844 & B lymphocytes, follicular DCs \\
\hline CD79a & 14.1 & $5,380.2$ & 381.6 & NM_007655 & B lymphocytes \\
\hline CD22 & 50.7 & $4,114.5$ & 81.2 & NM_009845 & B lymphocytes \\
\hline CD2 & 28.6 & $2,131.7$ & 74.5 & NM_013486 & Thymocytes, T and B lymphocytes, NK cells, myeloid cells, erythrocytes \\
\hline CD72 & 109.7 & $4,688.9$ & 42.7 & BC003824 & B lymphocytes, follicular DCs, subset of T lymphocytes \\
\hline CD22 & 8.0 & 249.4 & 31.2 & AF102134 & B lymphocytes \\
\hline CD79b & 254.5 & $7,414.6$ & 29.1 & NM_008339 & B lymphocytes \\
\hline CD1d2 & 5.1 & 114.1 & 22.4 & NM_007640 & Leukocytes \\
\hline CD83 & 138.8 & $2,262.7$ & 16.3 & NM_009856 & DCs, activated T lymphocytes \\
\hline CD38 & 293.2 & $3,917.3$ & 13.4 & BB256012 & B lymphocytes, activated T lymphocytes, subset of thymocytes \\
\hline CD1d1 & 123.6 & $1,228.9$ & 9.9 & NM_007639 & Leukocytes \\
\hline CD36 & 11.5 & 107.8 & 9.4 & AK004192 & Platelets, adipocytes, monocytes, macrophages, ECs, erythrocytes \\
\hline CD160 & 7.8 & 70.3 & 9.0 & AU045688 & T lymphocytes, NK cells \\
\hline CD3e & 151.6 & $1,222.5$ & 8.1 & NM_007648 & T lymphocytes, subset of thymocytes, NKT cells \\
\hline
\end{tabular}

Affymetrix arrays were used to compare gene expression profiles between BM cell populations. Genes shown had a minimum 5-fold difference in expression. Note the significantly higher level of CD44 expression in the CD44hi population versus CD44/o cells.

Transplanted CD44hi cells differentiate into microglia. Having better defined the population of active cells from BM, we next sought to characterize the cells that targeted to the retina after injection into the eye. CD44hi-injected retinas from the OIR model were analyzed by immunohistochemistry with antibodies to a variety of cellular markers. When studied at P12, immediately following the hyperoxia phase, we found many of the transplanted cells localized to the superficial (inner) layer of the avascular central retina (Figure 5A). Frequently, injected $\mathrm{GFP}^{+}$cells were observed to assume a branched morphology and localize between vessels (Figure 5B). Other transplanted cells were found in close association with the retinal vasculature (Figure 5, C-H). We were unable to detect expression of CD31 or NG2 on the transplanted BM cells, which suggests that these cells do not differentiate into endothelial cells or pericytes, respectively. Using $3 \mathrm{D}$ image reconstruction, we observed that the transplanted cells, while often residing in close proximity to the vessels, did not line the vessel lumen (Figure 5, H and I), further demonstrating that these cells were unlikely to differentiate into endothelial cells. Finally, injection of CD $44^{\text {hi }}$ BM cells isolated from mice expressing GFP under control of the endothelial Tie-2 promoter showed no GFP expression in the eye, again demonstrating a lack of endothelial differentiation. In contrast, the macrophage/microglia marker F4/80 labeled many $\mathrm{GFP}^{+}$cells in $\mathrm{CD}_{4} 4^{\text {hi }}$ treated eyes (Figure 5, C and D). Labeling with CD11b (Figure 5, $\mathrm{E}$ and $\mathrm{F}$ ) and isolectin GS-IB 4 (GS lectin) was also widely observed on the transplanted cells; these - together with F4/80 - are markers used to identify microglia in the CNS (21). In addition to the expression of these microglial markers, the transplanted CD44hi cells took on a morphology and localization indistinguishable from endogenous microglia (Figure 5, B, G, J, and K), which are known to associate with vessels as well as to localize within intervascular areas $(22,23)$. Thus, the myeloid progenitor population selected from BM appears to differentiate into microglia, the resident myeloid population of the retina. This pattern of differentiation was also observed when CD44hi cells were injected into normal neonatal eyes at P7 or injured adult eyes, indicating that ischemia is not the only signal that instructs these cells to differentiate and that developmental as well as injury cues may also have a role. Interestingly, this effect was not observed after injection into normal adult eyes.

Microglia participate in retinal vascularization. Based on the finding that transplanted BM-derived myeloid progenitor cells differentiated into microglia in the OIR model and promoted revascularization and repair of the central retina, we then asked whether microglia have a more generalized role in retinal vascularization. For the mouse model of OIR, the C57BL/6J mouse is the most widely used strain because it reliably provides significant vascular obliteration and preretinal vascular tuft formation (14). In contrast, the BALB/cByJ strain under the same conditions did not form preretinal tufts to an appreciable degree, and the central vaso-obliteration observed during hyperoxia very quickly revascularized following return to normoxia (Figure 6A). The underlying cause for these differences was not clear, so we tested the hypothesis that a difference in retinal microglia exists between 


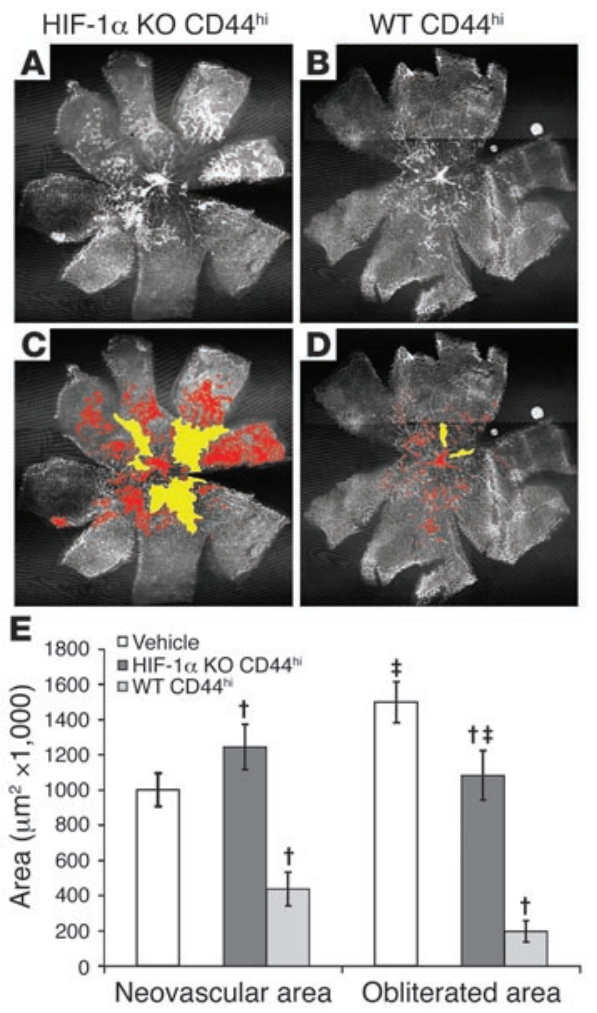

\section{Figure 4}

HIF-1 $\alpha$ expression is required for myeloid progenitors to mediate repair in the OIR model. (A and B) Representative GS lectin-stained retina whole mounts from eyes treated with CD44hi cells from myeloid-specific HIF-1 $\alpha$ knockout mice (A) or wild-type mice (B). (C and D) Quantified areas of vascular obliteration (yellow) and neovascular tufts (red) in retinas from $\mathbf{A}$ and $\mathbf{B}$. (E) Compiled data showing significant loss of repair activity in the eyes treated with $\mathrm{CD} 44^{\text {hi }}$ cells from HIF-1 $\alpha$ knockouts. ${ }^{\dagger} P \leq 0.0003,{ }^{\ddagger} P=0.024$. Values represent mean \pm SEM $(n=15)$. Statistics were generated using paired eyes. Magnification, $\times 4$.

these strains. Indeed, we found that microglia persisted in greater numbers within the vaso-obliterated central retina in BALB/cByJ mice over the course of 48 hours of ischemia/hypoxia compared with the $\mathrm{C} 57 \mathrm{BL} / 6 \mathrm{~J}$ mice (Figure 6, B and C). This raises the possibility that the BALB/cByJ strain is partially protected from retinopathy by the increased presence of endogenous retinal microglia during the repair phase. Similarly, transplantation of BM-derived myeloid progenitors into $\mathrm{C} 57 \mathrm{BL} / 6 \mathrm{~J}$ mice may be replacing and/or augmenting the function of the fewer endogenous microglia present in this strain, leading to a repair effect similar to that observed in the uninjected BALB/cByJ mice.

The microglia-associated differences described above in the OIR model suggest a role for these cells in retinal vascularization, but it is also possible that other strain-related differences could account for these results. We therefore sought to independently confirm these observations by examining whether microglia also play a role in normal retinal vascular development. This was accomplished by manipulating microglia numbers using clodronate-loaded liposomes, which are selectively taken up by, and induce apoptosis in, highly phagocytic macrophages/microglia (24-26). The specificity of microglial uptake in these experiments was demonstrated using labeled liposomes, which were found to colocalize exclusively

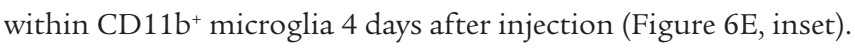
No labeled material was found in vascular cells or other CD11 $\mathrm{b}^{-}$ cells. Intravitreal injection of clodronate-loaded liposomes at P5 resulted in a significant reduction in the number of microglia and a dramatic disruption in the retinal vasculature when evaluated at P8. Massive capillary dropout was observed to correlate anatomically with areas of clodronate-mediated microglial cell death, suggesting that microglia are required for the development and/or maintenance of new vessels (Figure 6D). Similarly, injection of clodronate-loaded liposomes at $\mathrm{P} 2$ resulted in a $28 \%$ reduction in the area of vascularized retina $(n=6)$ at P6 compared with fellow eyes that received control PBS-loaded liposomes (Figure 6E). There appeared to be a difference in the density of the vascular networks as well, so we compared total vessel area, which was reduced by $41 \%$ with clodronate liposome treatment. Thus, microglia appear to be required for maintenance of immature vessels as well as for the growth of new vessels during retinal development.

\section{Discussion}

Decades of research in the field of angiogenesis have produced a much better understanding of the molecular mechanisms underlying this fundamental biological process (27). Effector molecules, their cellular receptors, intracellular signaling cascades and posttranscriptional regulators have been identified, and some have recently become the targets of compounds used clinically to inhibit pathologic angiogenesis associated with tumors and neovascular eye diseases. Understanding of cell-cell and cell-matrix interactions that affect angiogenesis at the tissue level has improved, and more recently a role for circulating cells in the regulation of vascular homeostasis in the adult organism has been identified in a number of organs, including the eye $(28,29)$. Our results suggest that cell-based therapy using adult BM-derived progenitor cells could have significant clinical utility in the treatment of ischemic retinopathies such as retinopathy of prematurity (ROP) and diabetes. One advantage of such an approach, in contrast to that of inhibiting angiogenesis with small molecules or recombinant proteins, lies in the ability of the cell to adapt and respond to a changing environment. Current therapeutic approaches to the treatment of neovascular eye diseases generally involve the application of a single angiostatic agent to the eye. Cell therapy likely involves numerous factors produced by the cell that can be appropriately modulated in response to changing conditions. This shift from antiangiogenics and destructive procedures that inhibit or ablate hypoxia-driven neovascularization to cell-based therapies that facilitate repair and stabilization may be of benefit in the treatment of certain ocular disorders.

Retinal microglia have historically been viewed as immunocompetent cells that respond to inflammation and infection, phagocytosing debris created during normal developmental remodeling or degenerative disease. A role for microglia in promoting retinal vascularization has not previously been clearly described. Here we present data demonstrating that adult BMderived cells expressing surface markers of myeloid progenitors differentiated into microglia and facilitated the enhanced recovery of vasculature after hypoxic injury. This process is HIF- $1 \alpha$ dependent and describes what we believe to be a novel role for myeloid progenitors in modulating angiogenesis. We extended these findings to show that endogenous microglia had an important role in promoting and maintaining retinal vascularization during normal development. 

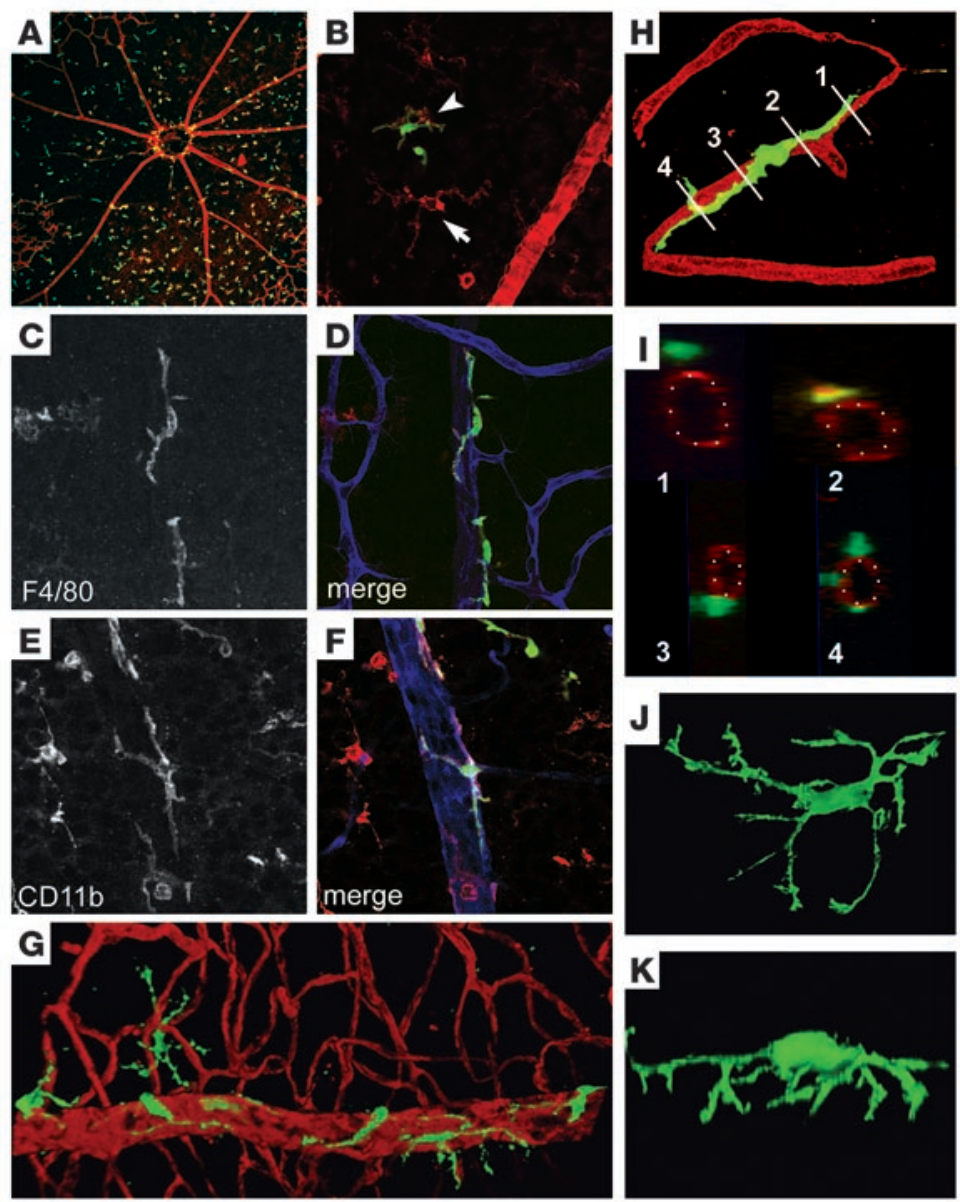

\section{Figure 5}

Intravitreally injected CD44 ${ }^{\text {hi }}$ cells take on characteristics of retinal microglia. (A) Transplanted CD44hi cells (green) injected at P7 localized to and survived within the avascular central retina at P12. Many cells labeled with GS lectin (red), which labels microglia in the retina, and thus appear yellow. (B) CD44hi cells (green, arrowhead) often assumed a ramified morphology similar to endogenous microglia. Endogenous microglia (arrow) and retinal vessels were stained with GS lectin (red). (C-F) In addition to the intervascular localization shown in B, transplanted CD44hi cells also assumed a perivascular localization and were positive for $F 4 / 80$ (C) and CD11b (E), markers of macrophages/microglia. D and $\mathbf{F}$ are merges with GFP expression (green), GS lectin (blue), and F4/80 (red, D) or CD11b (red, F). (G) CD44hi cells assumed both perivascular and intervascular localization (3D image). Note the ramified, microglia-like morphology of the GFP ${ }^{+}$cells. ( $H$ and I) $\mathrm{CD} 44^{\text {hi }}$ cells did not form any portion of the vessel lumen, as shown by $3 \mathrm{D}$ imaging. The image in $\mathbf{H}$ was rotated such that the vessel could be viewed in cross-section. (I) Numbered positions indicated in $\mathbf{H}$ show that the $\mathrm{GFP}^{+}$cell (green) was found on the outside of the CD31-labeled (dotted) endothelial lumen. ( $\mathbf{J}$ and $\mathbf{K}$ ) A single transplanted CD44hi cell (3D image) shown en face $(\mathbf{J})$ and in profile $(\mathbf{K})$ demonstrating the highly ramified morphology taken on by these cells after injection into the eye. Magnification, $\times 10(\mathbf{A}), \times 60(\mathbf{B}-\mathbf{I}), \times 120(\mathbf{J}$ and $\mathbf{K})$.
Our observation that transplanted myeloid progenitors dramatically enhanced revascularization of ischemic areas in the neonatal mouse retina while concomitantly reducing preretinal neovascular tuft formation is an apparent paradox. One explanation would be that the reduction in neovascular tuft formation is actually secondary to the accelerated physiologic revascularization of the central retina. Reports from other investigators have indicated that macrophages, under the influence of hypoxia, secrete factors that cause local endothelial proliferation resulting in tuft formation $(30,31)$. These macrophages are presumably recruited from the circulation and might migrate into the retina to phagocytose cellular debris resulting from oxygen-induced vaso-obliteration. When the animal is returned to normoxia, there may be a subpopulation of the macrophages that were distant enough from the blood supply to experience hypoxia, yet close enough to stimulate endothelial proliferation, thus contributing to the generation of neovascular tufts. Since they arise from the circulation, these macrophages would be concentrated near the remaining vasculature in the periphery. After injecting CD $44^{\text {hi }}$ myeloid progenitors into the OIR model, we observed large numbers of the transplanted cells localizing to the central ischemic retina where many endogenous microglia were lost. It may be that this localization replaces the function of lost microglia and establishes appropriate gradients of angiogenic factors that promote the controlled revascularization of the damaged areas. This idea is supported by our observations in the BALB/cByJ mouse in which the endogenous retinal microglia persisted in greater numbers after oxygen exposure and in which revascularization occurred at a rate similar to that of C57BL/6J mice treated with myeloid progenitors, with few or no tufts formed. Thus, faster vascular repair might reduce the overall hypoxia experienced by the retina and thereby reduce tuft formation. These "appropriate" gradients established by transplanted myeloid cells (in C57BL/6J mice) or endogenous microglia (in $\mathrm{BALB} / \mathrm{cByJ}$ mice) might also represent a stronger guidance signal, overriding that generated by the recruited macrophages, and promote functional physiologic revascularization. Alternatively, the injected BM cells may have an immunosuppressive effect on the endogenous macrophages that reduces the output of tuft-inducing angiogenic factors. These mechanisms, or a combination thereof, could plausibly account for the observed reduction in neovascular tuft formation.

The use of cell therapy to promote vascularization has been spearheaded by the field of cardiology with the goal of collateralizing ischemic myocardium and extremities. A substantial amount of evidence indicates that BM cells are effective at improving perfusion and cardiac function (32). However, it is not yet clear which cell type(s) are responsible for the observed effects. Previous work from our laboratory using models of retinal development and retinal degeneration demonstrated a vasculotrophic effect of injected BM-derived cells $(9,10)$. In these studies endothelial progenitor cells (EPCs) were observed to play an important role. In the present study using the OIR model, we have demonstrated that myeloid cells were the predominant mediator of the repair effects. This apparent discrepancy could be the result of one or 
Figure 6

Microglia participate in retinal vascularization. Comparing C57BL/6J and BALB/CByJ strains revealed a difference in the number of CD11 $b^{+}$microglia during the ischemic phase of OIR. (A) In whole mounts, both strains showed similar vaso-obliteration in the central retina at P12. However, at P17, there were dramatic differences in the vasculature, with C57BL/6J showing abundant neovascular tufts and little revascularization of the central retina. (B) C57BL/6J retinas contained fewer $\mathrm{CD} 11 \mathrm{~b}^{+}$ microglia over the course of 48 hours of ischemia compared with BALB/cByJ. The optic nerve head is positioned at the lower right in all images. (C) Quantification of CD11 b+ microglia over time shows that $\mathrm{C} 57 \mathrm{BL} / 6 \mathrm{~J}$ retinas had fewer microglia at P12 (0 hours) and less than half the number of microglia present in the retinas of BALB/cByJ mice at P14 (48 hours of ischemia). $P \leq 0.02$ for BALB/cByJ versus $\mathrm{C} 57 \mathrm{BL} / 6 \mathrm{~J}$ at all time points ( $n=8-11)$. (D) Microglia depletion in C57BL/6J induced dramatic loss of preexisting microvasculature during retinal development. Injection of clodronate liposomes at $\mathrm{P} 5$ resulted in significant loss of $\mathrm{CD} 11 \mathrm{~b}^{+}$microglia and capillary dropout at P8. Images depict similar locations in the central retina, with the optic nerve head at lower right. (E) Injection of clodronate liposomes into C57BL/6J at $\mathrm{P} 2$ caused significant depletion of CD11b+ microglia and dramatic disruption of the vasculature at P6 compared with control PBS liposome-treated fellow eye. Inset: Labeled liposomes (red) were taken up specifically by CD11b+ microglia (green) and not by GS lectin-labeled vascular cells (blue), nor any other CD11b- cells. Magnification, $\times 4$ (A and $\mathbf{E}$, top panels), $\times 10$ ( $\mathbf{D}$ and $\mathbf{E}$, bottom panels), $\times 20$ (B), $\times 60$ (E, inset).
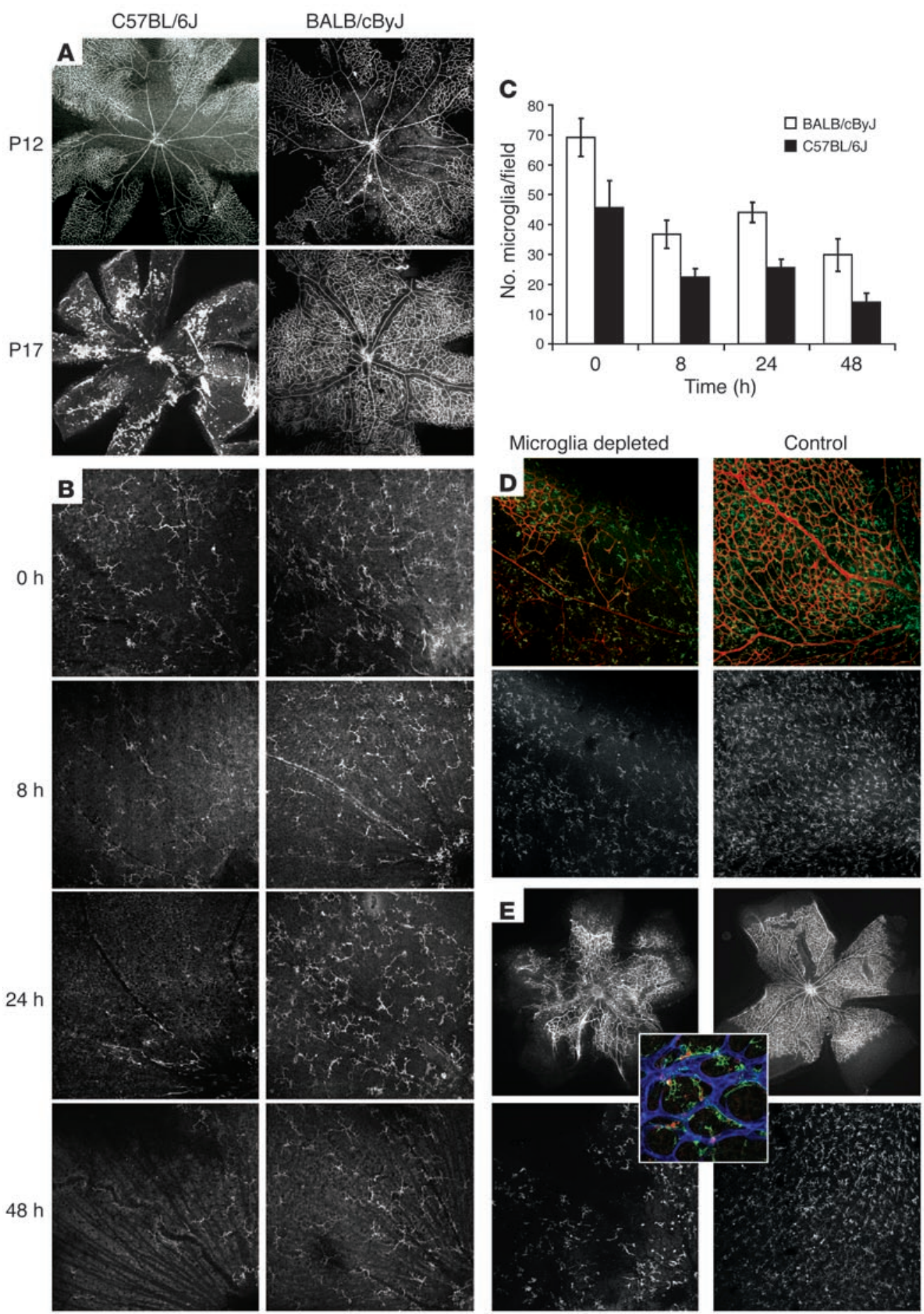

both of the following possibilities: (a) different subpopulations of $\mathrm{BM}$ cells are active under different conditions, or (b) in addition to EPCs, myeloid progenitors played an underappreciated role in our earlier findings. Numerous studies from other investigators have explored the potential role of BM-derived EPCs and have demonstrated that these cells are present in new or collateral vessels $(12,28,29)$. However, recent data suggest that heterogeneous $\mathrm{BM}$ populations such as mononuclear cells or unfractionated cells, which contain very small numbers of stem cells and/or EPCs, can also significantly enhance collateral development. This suggests that perivascular cells, as well as those directly incorporating into vessels, are likely to be involved (33). Most recently, Grunewald et al. (34) demonstrated an essential role for myeloid cells in adult neovascularization. This work demonstrated that myeloid cells recruited from the circulation in response to local VEGF expression in the adult heart or liver assumed a perivascular location and were retained there by stromal cell-derived factor 1 . It was further shown that the recruited myeloid cells promoted vessel growth in these organs in a paracrine fashion. Thus, a role is emerging for recruited myeloid cells in promoting vessel growth. 
The results of the present study may be the first step toward clinical use of specific subpopulations of autologous BM-derived progenitor cells in retinal vascular diseases such as ROP. We found that the neural retina and retinal vasculature appeared similar to uninjected retinas 6 months after injection and hyperoxia exposure, no tumors or teratomas were observed, and no extraocular $\mathrm{GFP}^{+}$cells were found in spleens or livers. These findings, along with the robust effect in the OIR model and the finding that beneficial effects (albeit partial) were observed even when cells were transplanted relatively late in the disease process, all support the possibility of clinical application. In very recent work, we have found that human $\mathrm{BM}$ contains a $\mathrm{CD} 44^{\mathrm{hi}}$ population with surface marker expression similar to that of mouse BM. We are currently testing the efficacy of these cells in OIR and other ocular disease models. One potential clinical application would involve the harvesting of umbilical cord blood cells during the birth of a high-risk premature infant, sorting of the appropriate cell population(s), and autologous intravitreal injection of these progenitor cells into the infant eye to prevent ROP. This approach might also be useful as a treatment for proliferative diabetic retinopathy and other adult ischemic retinopathies, where myeloid progenitors could be isolated from autologous BM and used to repair ischemic regions of the retina. Use of such a therapy could replace the repeated use of angiostatics in diabetic patients, as systemic exposure to these drugs could exacerbate ischemic conditions in other parts of the body as well as wound-healing problems that these patients often experience. Importantly, the nature of cell therapy, with continuous production of trophic factors over an extended period of time, would be expected to result in longer-term efficacy, potentially reducing the number and frequency of interventions required.

Further studies are necessary to assess the long-term efficacy and safety of using cell-based approaches for the treatment of retinal disease. Our data suggest that such an approach may be efficacious in the treatment of human diseases that most closely resemble OIR, such as ROP. The use of cell-based therapy, with its inherent ability to respond to microenvironmental cues with temporally and functionally complex actions, may provide a novel treatment for other ischemic retinopathies, and such potential applications are currently under study.

\section{Methods}

Animals and the OIR model. All experiments were performed in accordance with the NIH Guide for the Care and Use of Laboratory Animals, and all experimental procedures were approved by The Scripps Research Institute Animal Care and Use Committee. OIR was induced in C57BL/6J mice according to the protocol described by Smith et al. (14). For comparison, $\mathrm{BALB} / \mathrm{cByJ}$ mice were also subjected to the same conditions. Briefly, P7 pups and their mothers were transferred from room air to an environment of $75 \%$ oxygen for 5 days and afterward returned to room air. The hyperoxic environment was created and maintained using a chamber from BioSpherix. Under these conditions, large hypovascular areas formed in the central retina during hyperoxia in $\mathrm{C} 57 \mathrm{BL} / 6 \mathrm{~J}$ mice, and abnormal preretinal neovascularization occurred after return to normoxia, peaking at around P17 and ultimately resolving (Figure 1).

Cell preparation. Mouse BM cell isolation was performed as previously reported (9). Briefly, BM cells were harvested from femurs and tibias of transgenic mice expressing eGFP under control of an actin promoter (acteGFP) and were processed using 2 different methods. In the first method, mononuclear cells were separated by density gradient using Fico/Lite-LM (Atlanta Biologicals) and labeled with biotin-conjugated lineage antibod- ies (Mouse Lineage Antibody Cocktail; BD Biosciences - Pharmingen). This was followed by incubation with streptavidin or anti-biotin magnetic beads and sorting using the MACS cell sorting system (Miltenyi Biotech) to obtain the Lin-HSC population. In the second method, whole BM was incubated with a fluorescently labeled antibody directed against CD44 (BD Biosciences - Pharmingen, clone IM7). Fluorescence-activated cell sorting (FACS) was performed on a FACSAria (BD) and was used to isolate CD44hi and CD44lo subpopulations.

$B M$ cell characterization. Further analysis of the different cell subpopulations was performed using 2 methods: (a) 2-color flow cytometry and antibodies against various lineage and progenitor cell surface markers including CD11a, CD11b, Ly6G/C, F4/80, CD14, cKit (all from BD Biosciences - Pharmingen), and CD115 (AbD Serotec); and (b) mRNA expression analysis using Affymetrix Mouse Genome 4302.0 Arrays (Affymetrix) using standard methods described previously $(35,36)$. Gene expression was analyzed using GeneSpring software (version 4.1; Agilent Technologies).

Intravitreal injection. An eyelid fissure was created by gentle dissection to expose the globe in P2-P7 (prehyperoxia) mice. In 1 eye of each animal, 150,000 to $250,000 \mathrm{BM}$ cells in $0.5 \mu \mathrm{l}$ vehicle (Dulbecco's PBS containing $0.5 \%$ BSA and $2 \mathrm{mM}$ EDTA) were injected into the vitreous using a Hamilton syringe fitted with a 33-gauge needle (Hamilton). In the contralateral eye, an equal number of control cells or vehicle alone was injected; in some cases no injection was performed to observe the natural course of the model. A small yet statistically significant reduction in the area of prelaminar neovascularization was observed in these experiments following injection of vehicle alone compared with uninjected controls, suggesting a partial angiostatic effect of the injection itself (37). In subsets of experiments, injection of cells was performed at later ages, between P9 and P12. Clodronate-loaded liposomes and PBS-loaded liposomes were provided by N. van Rooijen (Vrije Universiteit, Amsterdam, The Netherlands) and were injected intravitreally undiluted at $0.5 \mu \mathrm{l}$ volume at P5 or P2. To show specificity of uptake, in some experiments liposomes were labeled with DiI (Vybrant dye; Invitrogen) prior to injection and visualized by confocal microscopy.

Immunohistochemistry and visualizing the vasculature. Retinas were harvested at various times for imaging of the vasculature and to localize and characterize injected and endogenous cells. Quantification of vascular obliteration and preretinal neovascular tufts was performed at P17. In some cases, animals were anesthetized, and intracardiac fluoresceinlabeled high-molecular weight dextran (FITC Dextran; Sigma-Aldrich) was injected prior to dissection of the retinas to visualize patent vessels. In most cases, immunohistological techniques to stain blood vessels and GFP-expressing cells were used as previously described $(9,11)$. Briefly, retinas were fixed in $4 \%$ PFA for 1 hour followed by blocking in $20 \%$ FBS $/ 20 \%$ NGS for 1 hour at room temperature. This was followed by overnight incubation with GS lectin conjugated to a fluorescent label to identify vessels (Invitrogen). Retinas were laid flat with radial-relaxing incisions to obtain whole-mount preparations. In order to characterize the injected cells, immunohistochemistry was used to identify the following cellular markers in subsets of eyes: F4/80 (CALTAG Laboratories) and CD11b (BD Biosciences - Pharmingen), macrophage markers; CD31, an endothelial cell marker (BD Biosciences - Pharmingen); and NG2, a pericyte marker (Chemicon International).

Imaging and image analysis. Images of the retinal vasculature were obtained using a Radiance2100 MP laser scanning confocal microscope (Bio-Rad; Zeiss). Quantification of vaso-obliteration and neovascularization was carried out as described previously (17). In brief, the area of vascular obliteration was measured by carefully delineating the avascular zones in the central retina of GS lectin-stained retinas and calculating the total area using Photoshop CS2 (Adobe) or Volocity software (version 3.0; 
Improvision). Similarly, the area of preretinal neovascularization (tufts) was calculated by using confocal images focused at the preretinal plane and selecting tufts based on pixel intensities (tufts label more brightly that normal vasculature). Selected regions were then summed to generate total area of neovascularization. Student's $t$ test was used to statistically compare the different experimental groups. The number of eyes included in each group (at least 6 and as many as 55) is shown in the figures. To quantify the effects of microglia depletion, the area of the retina bound by the vascular front was calculated and expressed as a percentage of the total area of each retina. In addition, as a measure of vascular density, vessel area was calculated as a percentage of total retinal area. We generated $3 \mathrm{D}$ images of retinal vasculature and injected $\mathrm{BM}$ cells by collecting a $z$-series of confocal images and rendering them into volumes using Volocity software. Microglia density was quantified by collecting confocal images of the superficial central retina using a $\times 20$ objective lens, including a quadrant of the optic nerve head as a point of reference. Three observers blinded to experimental group calculated the number of $\mathrm{CD} 11 \mathrm{~b}^{+}$cells in these images, and the resulting values from each image were averaged. The reported values represent the mean of these mean counts. For microglia analysis, $n=8-11$ for each condition.

ERG. Full-field ERGs were recorded in anesthetized mice following overnight dark adaptation using a Ganzfeld dome and a computerized system (Espion E2 ${ }^{2}$; Diagnosys). Pupils were dilated with $1 \%$ tropicamide and $2.5 \%$ phenylephrine (Fisher Scientific). Local anesthetic drops (benoxinate $\mathrm{HCl}$ $0.4 \%$; Fisher Scientific) were administered prior to positioning of gold loop electrodes on the cornea with $2 \%$ methylcellulose. Reference needle electrodes were placed below the eyes, and a ground needle electrode was placed subcutaneously near the tail. Rod and mixed cone-rod responses were recorded in the dark-adapted state to a series of white flashes of increasing intensities. In the light-adapted state (30 candelas per $\mathrm{m}^{2}$ background), cone responses to increasing intensities of white flashes at 1 and $16 \mathrm{~Hz}$ were recorded. All ERG responses were filtered at $0.3-500 \mathrm{~Hz}$, and signal averaging was used.

Statistics. Different experimental groups were compared using 1-tailed Student's $t$ test. Paired eyes were compared where indicated in figure legends. Results were considered statistically significant at $P<0.05$.

\section{Acknowledgments}

The authors thank the members of the Friedlander laboratory for helpful discussions concerning this work, with special thanks to Lea Scheppke, Valentina Marchetti, Kelcie King-York, and Ross Butschek for assistance in quantifications and Jeffrey Friedlander for long-term histological analyses. We thank The Scripps Research Institute Flow Cytometry Core Facility staff for their expert contributions to this work, Randall Johnson (UCSD, San Diego, California, USA) for providing the myeloid-specific HIF- $1 \alpha$ knockout mouse, and Nico van Rooijen (Vrije Universiteit, Amsterdam, The Netherlands) for providing the clodronate/PBS liposomes. This work was supported by grants EY11254 and EY14174 from the National Eye Institute, the Robert Mealy Program for the Study of Macular Degenerations, the V. Kann Rasmussen Foundation, the MacTel Foundation, and the Kovner Family Fund (to M. Friedlander). M.R. Ritter was supported by National Research Service Award F32 EY13916 from the National Eye Institute.

Received for publication July 13, 2006, and accepted in revised form September 26, 2006.

Address correspondence to: Martin Friedlander, The Scripps Research Institute, 10550 North Torrey Pines Road MB28, La Jolla, California 92037, USA. Phone: (858) 784-9138; Fax: (858) 784-9135; E-mail: friedlan@scripps.edu.

Matthew R. Ritter and Eyal Banin contributed equally to this work.

1. Das, A., and McGuire, P.G. 2003. Retinal and choroidal angiogenesis: pathophysiology and strategies for inhibition. Prog. Retin. Eye Res. 22:721-748.

2. Ryan, S.J., Hinton, D., Schachat, A., and Wilkinson, P. 2006. Retina. Elsevier. Philadelphia, Pennsylvania, USA. 3104 pP

3. Aiello, L.P. 2005. Angiogenic pathways in diabetic retinopathy. N. Engl. J. Med. 353:839-841.

4. Bressler, N.M., and Altaweel, M. 2005. Changes in retinal neovascularization after pegaptanib (Macugen) therapy in diabetic individuals. Ophthalmology. 113:23-28.

5. Jonas, J.B., Kreissig, I., and Degenring, R. 2005. Intravitreal triamcinolone acetonide for treatment of intraocular proliferative, exudative, and neovascular diseases. Prog. Retin. Eye Res. 24:587-611.

6. Mason, J.O., 3rd, et al. 2005. Visual outcome and risk factors for light perception and no light perception vision after vitrectomy for diabetic retinopathy. Am. J. Ophthalmol. 140:231-235.

7. Schmidt-Erfurth, U., Niemeyer, M., Geitzenauer, W., and Michels, S. 2005. Time course and morphology of vascular effects associated with photodynamic therapy. Ophthalmology. 112:2061-2069.

8. Van Wijngaarden, P., Coster, D.J., and Williams, K.A. 2005. Inhibitors of ocular neovascularization: promises and potential problems. JAMA. 293:1509-1513.

9. Otani, A., et al. 2002. Bone marrow-derived stem cells target retinal astrocytes and can promote or inhibit retinal angiogenesis. Nat. Med. 8:1004-1010.

10. Otani, A., et al. 2004. Rescue of retinal degeneration by intravitreally injected adult bone marrow-derived lineage-negative hematopoietic stem cells. J. Clin. Invest. 114:765-774. doi:10.1172/ JCI200421686.
11. Dorrell, M.I., Otani, A., Aguilar, E., Moreno, S.K., and Friedlander, M. 2004. Adult bone marrowderived stem cells use R-cadherin to target sites of neovascularization in the developing retina. Blood. 103:3420-3427.

12. Kocher, A.A., et al. 2001. Neovascularization of ischemic myocardium by human bone-marrowderived angioblasts prevents cardiomyocyte apoptosis, reduces remodeling and improves cardiac function. Nat. Med. 7:430-436.

13. Orlic, D., et al. 2001. Bone marrow cells regenerate infarcted myocardium. Nature. 410:701-705.

14. Smith, L.E., et al. 1994. Oxygen-induced retinopathy in the mouse. Invest. Ophthalmol. Vis. Sci. 35:101-111.

15. Connolly, S.E., Hores, T.A., Smith, L.E., and D'Amore, P.A. 1988. Characterization of vascular development in the mouse retina. Microvasc. Res. 36:275-290.

16. Dorrell, M.I., Aguilar, E., and Friedlander, M. 2002. Retinal vascular development is mediated by endothelial filopodia, a preexisting astrocytic template and specific R-cadherin adhesion. Invest. Ophthalmol. Vis. Sci. 43:3500-3510.

17. Banin, E., et al. 2006. T2-TrpRS inhibits preretinal neovascularization and enhances physiological vascular regrowth in OIR as assessed by a new method of quantification. Invest. Ophthalmol. Vis. Sci. 47:2125-2134

18. Semenza, G.L. 2003. Targeting HIF-1 for cancer therapy. Nat. Rev. Cancer. 3:721-732.

19. Clausen, B.E., Burkhardt, C., Reith, W., Renkawitz, R., and Forster, I. 1999. Conditional gene targeting in macrophages and granulocytes using LysMcre mice. Transgenic Res. 8:265-277.

20. Cramer, T., et al. 2003. HIF-1alpha is essential for myeloid cell-mediated inflammation. Cell. 112:645-657.

21. Guillemin, G.J., and Brew, B.J. 2004. Microglia, macrophages, perivascular macrophages, and pericytes: a review of function and identification. J. Leukoc. Biol. 75:388-397.

22. Hume, D.A., Perry, V.H., and Gordon, S. 1983. Immunohistochemical localization of a macrophage-specific antigen in developing mouse retina: phagocytosis of dying neurons and differentiation of microglial cells to form a regular array in the plexiform layers. J. Cell Biol. 97:253-257.

23. Provis, J.M., Penfold, P.L., Edwards, A.J., and van Driel, D. 1995. Human retinal microglia: expression of immune markers and relationship to the glia limitans. Glia. 14:243-256.

24. Van Rooijen, N., and Sanders, A. 1994. Liposome mediated depletion of macrophages: mechanism of action, preparation of liposomes and applications. J. Immunol. Methods. 174:83-93.

25. Schmidt-Weber, C.B., et al. 1996. Apoptotic cell death in activated monocytes following incorporation of clodronate-liposomes. J. Leukoc. Biol. 60:230-244.

26. Cursiefen, C., et al. 2004. VEGF-A stimulates lymphangiogenesis and hemangiogenesis in inflammatory neovascularization via macrophage recruitment. J. Clin. Invest. 113:1040-1050. doi:10.1172/JCI200420465.

27. Kerbel, R., and Folkman, J. 2002. Clinical translation of angiogenesis inhibitors. Nat. Rev. Cancer. 2:727-739.

28. Grant, M.B., et al. 2002. Adult hematopoietic stem cells provide functional hemangioblast activity during retinal neovascularization. Nat. Med. 8:607-612. 
29. Espinosa-Heidmann, D.G., Caicedo, A., Hernandez, E.P., Csaky, K.G., and Cousins, S.W. 2003. Bone marrow-derived progenitor cells contribute to experimental choroidal neovascularization. Invest. Ophthalmol. Vis. Sci. 44:4914-4919.

30. Ishida, S., et al. 2003. VEGF164-mediated inflammation is required for pathological, but not physiological, ischemia-induced retinal neovascularization. J. Exp. Med. 198:483-489.

31. Davies, M.H., Eubanks, J.P., and Powers, M.R. 2006. Microglia and macrophages are increased in response to ischemia-induced retinopathy in the mouse retina. Mol. Vis. 12:467-477.

32. Laflamme, M.A., and Murry, C.E. 2005. Regenerating the heart. Nat. Biotechnol. 23:845-856.

33. Kinnaird, T., Stabile, E., Burnett, M.S., and Epstein, S.E. 2004. Bone-marrow-derived cells for enhancing collateral development: mechanisms, animal data, and initial clinical experiences. Circ. Res. 95:354-363.

34. Grunewald, M., et al. 2006. VEGF-induced adult neovascularization: recruitment, retention, and role of accessory cells. Cell. 124:175-189.

35. Dorrell, M.I., Aguilar, E., Weber, C., and Friedlander, M. 2004. Global gene expression analysis of the developing postnatal mouse retina. Invest. Ophthalmol. Vis. Sci. 45:1009-1019.

36. Ritter, M.R., Dorrell, M.I., Edmonds, J., Friedlander, S.F., and Friedlander, M. 2002. Insulin-like growth factor 2 and potential regulators of hemangioma growth and involution identified by large-scale expression analysis. Proc. Natl. Acad. Sci. U. S. A. 99:7455-7460.

37. Penn, J.S., et al. 2006. Angiostatic effect of penetrating ocular injury: role of pigment epithelium-derived factor. Invest. Ophthalmol. Vis. Sci. 47:405-414.

38. Pahl, H.L., Rosmarin, A.G., and Tenen, D.G. 1992 Characterization of the myeloid-specific CD11b promoter. Blood. 79:865-870.

39. Fleming, T.J., Fleming, M.L., and Malek, T.R. 1993. Selective expression of Ly-6G on myeloid lineage cells in mouse bone marrow. RB6-8C5 $\mathrm{mAb}$ to granulocyte-differentiation antigen $(\mathrm{Gr}-1)$ detects members of the Ly-6 family. J. Immunol.
151:2399-2408.

40. Malorny, U., Michels, E., and Sorg, C. 1986. A monoclonal antibody against an antigen present on mouse macrophages and absent from monocytes. Cell Tissue Res. 243:421-428.

41. Landmann, R., Wesp, M., and Obrecht, J.P. 1991. Cytokine regulation of the myeloid glycoprotein CD14. Pathobiology. 59:131-135.

42. Junttila, I., Bourette, R.P., Rohrschneider, L.R., and Silvennoinen, O. 2003. M-CSF induced differentiation of myeloid precursor cells involves activation of PKC-delta and expression of Pkare. J. Leukoc. Biol. 73:281-288.

43. Kina, T., et al. 2000. The monoclonal antibody TER-119 recognizes a molecule associated with glycophorin A and specifically marks the late stages of murine erythroid lineage. Br. J. Haematol. 109:280-287.

44. Coffman, R.L. 1982. Surface antigen expression and immunoglobulin gene rearrangement during mouse pre-B cell development. Immunol. Rev. 69:5-23. 